\title{
OFERTA ACADÉMICA DE LA URACCAN EN EL NIVEL DE GRADO PARA EL QUINQUENIO 2014-2018
}

\author{
Letisia Castillo ${ }^{[1]}$ \\ Leonor Ruiz ${ }^{[2]}$ \\ Marlon Peralta ${ }^{[3]}$ \\ Alba Luz Dávila ${ }^{[4]}$ \\ Ivania Simons ${ }^{[5]}$ \\ Juan Francisco Perera Lumbíi ${ }^{[6]}$
}

\section{Resumen}

Esta investigación tuvo como propósito adecuar la oferta académica de la URACCAN, basada en las realidades y necesidades de formación profesional en el nivel de grado de la Costa Caribe de Nicaragua para el quinquenio 2014-2018. Se desarrolló en las Regiones Autónomas, específicamente en los municipios donde la Universidad tiene presencia (Bluefields, Laguna de Perlas, Nueva Guinea, El Coral, Almendro, San Miguelito, Siuna, Rosita, Bonanza, Waslala, Bilwi y Waspam). Se trabajó con los estudiantes finalistas de Secundaria (undécimo grado) de todos los centros educativos, incluyendo a los directores de estos colegios. Asimismo, se entrevistaron a autoridades territoriales, gobiernos municipales y regionales. Participaron en el estudio los principales empleadores de nuestros graduados/graduadas de las diferentes carreras, los que dirigen las principales instituciones de gobierno regional, municipal y central, así como el análisis de la Estrategia de Desarrollo de la Costa Caribe y el Plan Nacional de Desarrollo del País.

Las carreras más demandadas para ser ofertadas e implementadas en los cuatro Recintos son: Medicina Intercultural, Ingeniería Civil, Ingeniería en Sistema, Derecho con énfasis en Derecho Autonómico y Administración de Empresas. La oferta académica de la Universidad cubre algunas áreas en la formación de profesionales de la estrategia en desarrollo de la Costa Caribe; pero, otras quedan pendientes como: Ordenamiento territorial, seguridad alimentaria, agua y saneamiento, convivencia comunitaria y administración de justicia, protección de los bosques y los ecosistemas, transporte (canal intercostero, construcción de carreteras), energía renovable y agua (hidroeléctricas) y agroforestería comunitaria, por lo que estas deben ser parte fundamental en la nueva oferta académica que llevará a cabo la Universidad en el próximo quinquenio.

Palabras clave: oferta académica, formación profesional, demanda académica.

[1] Máster en Docencia Universitaria, Directora Académica de URACCAN

[2] Máster en Antropología, Secretaria Académica de URACCAN Las Minas

[3] Licenciado en Informática, Secretario Académica de URACCAN Nueva Guinea

[4] Máster en Pedagogía, Secretaria Académica de URACCAN Bluefields

[5] Máster en Docencia Universitaria, Secretaria Académica de URACCAN Bilwi

[6] Máster en Ciencias Forestales, Director de Investigación y Posgrado de URACCAN 


\section{Summary}

This research was aimed to adjust URACCAN academic offer, based on the realities and needs of professional training in the degree level of the Caribbean Coast of Nicaragua for a period of five years (2014 to 2018). The research was developed in the Autonomous Regions, specifically in the municipalities where the University is present (Bluefields, Pearl Lagoon, Nueva Guinea, El Coral, Almendro, San Miguelito, Siuna, Rosita, Bonanza, Waslala, Bilwi and Waspam). We worked with the finalists high school students (eleventh grade) of all the schools, including the directors of these schools. Likewise, interviewed were applied to authorities of the territorial, municipal and regional governments. The major employers who run the principal institutions of the central, regional and municipal government participated in the study of our graduates from different careers, making an analysis of the development strategy of the Caribbean Coast and the Country National Development Plan.

The most demanded careers to be offered and implemented in the four campuses are: Intercultural Medicine, Civil Engineering, System Engineering, and Law with emphasis on Autonomy Law and Business Administration. The academic offer of the University covers some areas in the professional training of development strategy in the Caribbean Coast, but they are others that remain pending as: Land management, food security, water and sanitation, community life and administration of justice, protection of forests and ecosystems, transportation (intercoastal canal or waterway, road construction), renewable energy and water (hydroelectric power) and community agroforestry; so these should be a fundamental part of the new academic offer that the University will carry out during the next five years.

Keywords: Academic offer, professional training, academic demand.

\section{Introducción}

La Conferencia Mundial de Educación Superior (CMES) realizada en 1998, acordó la "Declaración Mundial sobre la Educación Superior en el siglo XXI: visión y acción". La cual expone ampliamente la importancia de un involucramiento de la universidad con la sociedad en una perspectiva de largo plazo y plantea la pertinencia de la Educación Superior como la respuesta a las necesidades y expectativas de la sociedad, servir a esa sociedad en procura de "erradicar la pobreza, la intolerancia, la violencia, el analfabetismo, el hambre, el deterioro del medio ambiente y las enfermedades, principalmente mediante un planteamiento interdisciplinario y transdisciplinario para analizar los problemas", contribuir en la construcción de una nueva sociedad que excluya las prácticas violentas, vinculada también con el mundo del trabajo y todos los demás sectores sociales ${ }^{[7]}$. 
La evaluación de la pertinencia de los programas educativos alude al análisis de la capacidad de la Universidad para responder a las expectativas de la comunidad. En términos más amplios, estudia aspectos referentes a las prioridades establecidas por los planes de desarrollo; estudios de oferta y demanda educativa; y estudios de competencia y de formación integral del estudiantado, así como de los mecanismos de producción y distribución del conocimiento.

La Universidad de las Regiones Autónomas de la Costa Caribe Nicaragüense en su compromiso con la sociedad costeña de brindar y desarrollar una educación de calidad y pertinente a las necesidades de los pueblos indígenas, afrodescendientes y mestizos, se propone avanzar en la tarea de analizar la cobertura, atención y demandas educativas, así como de extensión social comunitaria. Lo que responde a la necesidad permanente de ajuste entre la oferta académica por parte de la Universidad y la demanda real que recibe. Ésta es una tarea permanente de toda universidad pública que se ve obligada a cumplir una misión social con el mejor uso posible de los recursos.

\section{Revisión de literatura}

La universidad debe hacer un esfuerzo por entender las señales de la sociedad y si la sociedad cambia continuamente, debe responder a las necesidades de formación como parte de su pertinencia. Tünnermann define ser pertinente: "el estar en contacto con las políticas, con el mundo del trabajo, con los demás niveles del sistema educativo, con la cultura y las culturas, con los estudiantes y profesores, con todos, siempre y en todas partes" (2006:7).

Por tanto, implica ser consciente y estar en contacto con las características y necesidades del contexto en el que se está desarrollando cualquier actividad, desde sus diversos ámbitos; social, educativo, económico, político y normativo. En el campo de la educación la pertinencia responde a preguntas: ¿Quién o quiénes determinan o definen el sentido y el contenido del curriculum? ¿Cómo identificar que elementos hay que tomar en cuenta para describir la pertinencia?. Identificar la pertinencia educativa implica, sin duda alguna y en primera instancia estar en contacto con la realidad contextual, pues es ahí donde se desarrolla y concretiza el currículum.

Según Rojo (1999), la pertinencia es la adecuada interpretación de problemas y necesidades de la sociedad y al mismo tiempo una clara visión de lo que debe ser su accionar futuro. Por otro lado, Tunnerman (2000) opina que la pertinencia también está en función del quehacer de las IES a partir de las necesidades sociales.

En estas definiciones hay coincidencias básicas; no obstante, una propuesta que centra los fines de este trabajo es la de Herrera y colaboradores (2005), que la reconocen como el grado en que la Educación Superior (ES) responde a problemas, demandas y expectativas de la sociedad, para preservar y fortalecer la cultura y los 
valores; además de formar recursos humanos acordes con los procesos económicos, políticos y sociales, y desarrollar conocimiento científico y aplicaciones tecnológicas.

En este sentido, la oferta educativa es dependiente de la demanda educativa, formada por las necesidades y requerimientos educativos de la sociedad; asimismo de factores externos, como la estructura demográfica, la estructura educativa, las políticas del Estado, los intereses y valores individuales. También de factores internos como los planes, políticas y situaciones de los programas en las propias instituciones educativas (Alejo et al, 2009).

Nuestro modelo de universidad comunitaria intercultural, establece que la finalidad de la Educación Superior de pueblos indígenas y comunidades étnicas debe ser realista y práctica, reflejar la riqueza espiritual de las cosmogonías y de las filosofías indígenas, fuentes de sabiduría y de equilibrio armónico del ser humano con su tierra. Por tanto, debe ser estructurada sobre la transmisión de sistemas integrados de conocimiento holístico y enraizarse en la fuerza viva de la espiritualidad indígena, que pueda permitir el enriquecimiento de valores éticos y humanísticos (URACCAN, 2004 a).

Así mismo, dicho modelo plantea como retos, en primera instancia el cambio de visiones del personal docente universitario de forma que facilite adquirir no sólo habilidades para el mercado laboral, sino también actitudes de respeto, práctica intercultural verdadera y valores éticos congruentes con la filosofía ancestral de los pueblos indígenas y comunidades étnicas. De igual forma, la vinculación de los subsistemas educativos formales (primaria, secundaria y educación técnica). También la construcción de espacios de concertación y búsqueda conjunta de soluciones a los principales problemas integrales que la sociedad multicultural (URACCAN, 2004).

\section{Materiales y método}

La investigación se desarrolló en las Regiones Autónomas, específicamente en los municipios donde la Universidad tiene presencia: (Bluefields, Laguna de Perlas, Nueva Guinea, El Coral, Almendro, San Miguelito, Siuna, Rosita, Bonanza, Waslala, Bilwi y Waspam).

\section{Tipo de Investigación}

La investigación fue del tipo mixta, debido a que se desarrollaron tanto los enfoques cualitativos y cuantitativos. Según Sampieri (2006). En las investigaciones mixtas los dos enfoques (cualitativo y cuantitativo), son paradigmas de la investigación científica, pues ambos emplean procesos cuidadosos, sistemáticos y empíricos en su esfuerzo por generar conocimientos y utilizan en general, cinco fases similares y relacionadas entre sí. 
- Llevan a cabo observación y evaluación de fenómenos.

- Establecen posiciones o ideas como consecuencia de la observación y evaluación realizadas.

- Demuestran el grado en que las suposiciones o ideas tienen fundamento.

- Revisan tales suposiciones o ideas sobre la base de las pruebas o del análisis.

- Proponen nuevas observaciones y evaluaciones para esclarecer, modificar y fundamentar las suposiciones e ideas; o incluso para generar otras.

La información que generó esta investigación es de los siguientes tipos:

- Cuantitativa, respecto a la demanda de estudios universitarios, la matrícula, el tipo de formación demandada, la oferta de titulaciones, la oferta de plazas.

- Cualitativa, respecto a las características de los estudios que demandan los estudiantes, empleadores, gobiernos municipales, territoriales, regionales y las necesidades de formación según el Plan Nacional de Desarrollo y el de la Costa Caribe nicaragüense.

\section{Población y grupos seleccionados}

Se investigo conjuntamente con los estudiantes finalistas de Secundaria (undécimo grado) en todos los centros educativos de las ciudades y comunidades donde la Universidad tiene presencia, incluyendo a los directores de estos colegios. Asimismo, se entrevistaron a autoridades territoriales, gobiernos municipales y regionales.

También participaron en el estudio, los principales empleadores de nuestros graduados/graduadas en las diferentes carreras, así como los que dirigen las principales instituciones de gobierno, regional, municipal y central.

\section{Muestra y tipo de muestreo}

Según Triola (2004) para el cálculo de la muestra en poblaciones finitas, se utilizó la siguiente fórmula:

$$
\begin{aligned}
& n=\frac{\left(Z_{\alpha / 2}\right)^{2}(p)(q)(N)}{\left(e^{2}\right)(N-1)+\left(Z \alpha_{/ 2}\right)^{2}(p)(q)} \\
& n=\frac{\left(1.96^{2}\right)(0.5)(0.5)(746)}{\left(0.03^{2}\right)(746-1)+\left(1.96^{2}\right)(0.5)(0.5)}
\end{aligned}
$$


En la fórmula anterior, se utilizó una proporción del 50\%, debido a que no se conocen datos anteriores sobre el mismo.

La muestra se dividió por estratos, ya que según Sampieri (2006), lo que aquí se hace es dividir a la población en sub poblaciones o estratos, y se selecciona una muestra para cada estrato. La estratificación aumenta la precisión de la muestra e implica el uso deliberado de diferentes tamaños de muestra para cada estrato, "A fin de lograr reducir la varianza de cada unidad de la media muestral". Posteriormente en la aplicación de las encuestas se desarrollo un muestreo aleatorio sistemático. Por lo que en el siguiente cuadro se detallan la muestra por colegio.

Cuadro No. 1. Municipios y el número de informantes por las técnicas

\begin{tabular}{|l|r|r|r|r|r|}
\hline \multicolumn{1}{|c|}{ Municipio } & \multicolumn{7}{|c|}{ Técnicas } & Encuestas \\
\hline Estudiantes & \multicolumn{2}{|c|}{ Organizaciones } & \multicolumn{1}{|c|}{ ONG } & Instituciones & $\begin{array}{c}\text { Gobiernos } \\
\text { Territoriales }\end{array}$ \\
\hline Siuna & 15 & 10 & 10 & 0 & 200 \\
\hline Rosita & 15 & 6 & 10 & 2 & 100 \\
\hline Bonanza & 10 & 5 & 10 & 1 & 80 \\
\hline Waslala & 10 & 5 & 10 & 0 & 80 \\
\hline Bluefields & 10 & 5 & 10 & 2 & 150 \\
\hline Laguna de & 5 & 5 & 5 & 0 & 25 \\
Perlas & 0 & 0 & 0 & 0 & 25 \\
\hline Wawashang & 18 & 10 & 15 & 3 & 250 \\
\hline Bilwi & 8 & 10 & 10 & 2 & 100 \\
\hline Waspam & 10 & 5 & 25 & 0 & 250 \\
\hline $\begin{array}{l}\text { Nueva } \\
\text { Guinea }\end{array}$ & $\mathbf{1 0 1}$ & $\mathbf{6 1}$ & $\mathbf{1 0 5}$ & $\mathbf{1 0}$ & $\mathbf{1 2 6 0}$ \\
\hline TOTAL & & & & & \\
\hline
\end{tabular}

Con las y los directores de colegio y empleadores, se tomó en consideración los siguientes criterios de inclusión:

- Empleadores activos de los graduados/as de la universidad en las diferentes carreras.

- Colaboradores activos con los Recintos.

- Líderes y lideresas religiosos, políticos, comunitarios, territoriales. 


\section{Variables/descriptores del estudio}

La investigación por ser mixta, se trabajaron las siguientes variables y descriptores:

- Oferta de grado de las diferentes universidades en la RAAS y RAAN.

- Demanda histórica de oferta académica 2002-2012.

- Comportamiento estudiantil en la admisión de las diferentes carreras

- Pertinencia de la oferta académica actual en base a las necesidades de desarrollo de la Costa Caribe nicaragüense y el país.

-Objetivo general de las carreras.

- Perfil de egreso.

- Potencial de desarrollo de la C.C.

- Demandas de formación con pertinencia cultural de empleadores y gobierno territorial, municipal, regional y nacional.

- Demandas de formación de empleadores.

- Demandas de formación de gobierno territorial.

- Demandas de formación de gobierno municipal.

- Demandas de formación gobierno regional.

- Demanda de formación gobierno nacional.

- Demanda de formación profesional de los futuros bachilleres de la Costa Caribe nicaragüense.

- Demandas de formación profesional de estudiantes del undécimo grado de secundaria.

\section{Técnicas e Instrumentos}

Se aplicaron encuestas a los estudiantes finalistas de secundarias. Entrevistas a empleadores, lideres/lideresas, directores de colegio. Así mismo, la revisión documental de informes y del plan de desarrollo de la Costa Caribe.

\section{Procesamiento y análisis de la información}

El procesamiento de las encuestas se realizó mediante el programa SPSS. Las entrevistas se transcribieron y analizaron en matrices, según las variables y descriptores del estudio. 


\section{Resultados y discusión}

\section{Diagnóstico de la oferta de grado de las diferentes Universidades en la Costa Caribe nicaragüense}

En base al mapeo realizado en los cuatro Recintos de la URCACAN, se encontró que tenemos presencia de tres Universidades: Martin Lutero (UML), Bluefields Indian and Caribean University (BICU) y Universidad de Occidente (UDO). Esta última, actualmente no tiene presencia en la región.

Cabe mencionar que según diagnóstico realizado por el Consejo Nacional de Universidades (CNU) en el año 2004, en la Región solo había presencia de la URACCAN y BICU, lo que se infiere que posterior a esta fecha es que se tuvo la presencia de las universidades antes mencionadas.

De acuerdo al área de conocimiento, en el cuadro siguiente, se puede apreciar, las carreras que han ofertado hasta el 2012. La comparación con la URACCAN únicamente es de acuerdo a la oferta de las otras universidades.

Cuadro No. 2. Carreras ofertadas de acuerdo al área de conocimiento de las diferentes universidades con presencia en la Costa Caribe nicaragüense

\begin{tabular}{|l|r|r|r|r|}
\hline \multicolumn{1}{|c|}{$\begin{array}{c}\text { ÁREA DEL } \\
\text { CONOCIMIENTO }\end{array}$} & URACCAN & MARTIN LUTERO & \multicolumn{1}{|c|}{ BICU } & UDO \\
\hline $\begin{array}{l}\text { Ciencias de la } \\
\text { educación }\end{array}$ & 11 & 3 & 8 & 0 \\
\hline Humanidades & 4 & 4 & 1 & 1 \\
\hline $\begin{array}{l}\text { Ciencias de la } \\
\text { Salud }\end{array}$ & 3 & 2 & 4 & 3 \\
\hline $\begin{array}{l}\text { Ciencias ad- } \\
\text { ministrativas e } \\
\text { informática }\end{array}$ & 3 & & 4 & 0 \\
\hline $\begin{array}{l}\text { Recursos natu- } \\
\text { rales y medio } \\
\text { ambiente }\end{array}$ & 1 & & 1 (Técnico \\
\hline $\begin{array}{l}\text { Tecnología y } \\
\text { construcción }\end{array}$ & 0 & & Superior) & \\
\hline $\begin{array}{l}\text { Ingenierías tec- } \\
\text { nológicas }\end{array}$ & & & 2 & 0 \\
\hline
\end{tabular}

Otro aspecto a tomar en cuenta, es que existen carreras que son comunes en las tres universidades mencionadas como: Educación Física, Enfermería, Licenciatura en Contabilidad Pública y Auditoría, Licenciatura en Ingles y Licenciatura en Pedagogía. 


\section{Demanda histórica de las carreras ofertadas por la universidad URACCAN}

Como punto de partida, analizamos las tendencias y el comportamiento de la oferta académica y la demanda de ingreso a la URACCAN en la última década.

Durante el período del 2002 al 2012, se han ofertado veintiséis carreras, de las cuales, diez carreras han sido las más demandadas históricamente. Para ello se tomo en cuenta los rangos entre 1931-400 estudiantes, se obtuvieron los siguientes resultados:

1) Informática Administrativa.

2) Ingeniería Agroforestal.

3) Licenciatura en Administración de Empresas.

4) Licenciatura en Sociología con mención en Autonomía.

5) Ingeniería en Zootecnia.

6) Licenciatura en Ciencias Sociales con mención en Desarrollo Local.

7) Licenciatura en Ciencias de la Educación mención en español.

8) Licenciatura en Ciencias de la Educación mención en Biologí.a

9) Licenciatura en Contabilidad Pública y Auditoría.

10) Licenciatura en Pedagogía.

Si nos detenemos a observar el crecimiento de la demanda en la Universidad en el período 2002 al 2012 por las carreras más demandadas, nos damos cuenta que ha habido un incremento significativo en siete carreras: Licenciatura en Administración de Empresas, Ingeniería Agroforestal, Licenciatura en Sociología con mención en Autonomía, Licenciatura en Pedagogía, Licenciatura en Contabilidad Pública y Auditoría, Licenciatura en Ciencias de la Educación con mención en Biología y Licenciatura en Informática Administrativa.

Por el contrario, las que tuvieron un decremento en la demanda son tres: Licenciatura en Ciencias Sociales con mención en Desarrollo Local, Ingeniería en Zootecnia y Licenciatura en Ciencias de la Educación con mención en español, es decir, después de 10 años, llegan menos jóvenes a solicitar un espacio en éstas (cuadro 3).

Si analizamos la matrícula con respecto a la variable sexo de manera general, se puede apreciar que durante el período investigado, ha prevalecido el sexo femenino con un $58 \%$ y para el masculino el $42 \%$. En este sentido, la matrícula femenina es superior a la masculina, la que se caracteriza por un crecimiento progresivo de la participación femenina en todas las áreas de estudio terciarias. Lo que coincide con UDUAL (2007) que al comenzar a masificarse el acceso a la Educación Superior el 
tradicional movimiento estudiantil latinoamericano de carácter urbano, masculino y de estudiantes no trabajadores desapareció como realidad social.

El comportamiento global ha sido que todas las carreras de Ciencias de la Educación, a excepto de Matemáticas, la mayoría pertenecen al sexo femenino, de igual manera para las Ciencias Económicas y Tecnológicas.

Con respecto a la variable de análisis etnia, se puede observar primeramente según los resultados que la Universidad URACCAN abre los espacios para el acceso de estudiantes de las diferentes etnias que conviven en la Costa Caribe nicaragüense, por lo que en esta ultima década las aulas de clases han sido espacios de una verdadera multiculturalidad. Sin embargo, ha habido etnias que en presencia estudiantil han predominado como el mestizo, seguido de los miskitus, creoles, mayangnas, garífunas y ramas.

Al tomar en cuenta la variable procedencia (Urbano-Rural) de los y las estudiantes que ingresaron a URACCAN en el período del 2002 al 2012, los datos nos refleja que prevalece los que proceden del área urbana, lo que hace para la Universidad un gran desafío de trabajo para que puedan acceder y permanecer estudiantes del área rural.

Podemos inferir, que la creación de carreras en este período (2002-2012) ha posibilitado la reorientación de la demanda, es decir, que la diversificación de la oferta mediante nuevas carreras permitiría que la demanda dejara de concentrarse en algunas carreras ya saturadas como Ingeniería Agroforestal, Licenciatura en Administración de Empresas y Licenciatura en Sociología con mención en Autonomía. Sin embargo, lo que observamos es que esto ha sucedido sólo en algunos casos, por ejemplo, en Medicina Intercultural y Licenciatura en Psicología en Contextos Multiculturales, en donde las carreras de reciente creación sí han visto atraída la demanda hacia ellas.

Demanda de formación profesional de los futuros bachilleres, empleadores, gobierno territorial, municipal, regional y nacional de la Costa Caribe nicaragüense. Al preguntarles a los futuros bachilleres de la Costa Caribe nicaragüense, si conocen las carreras que oferta la URACCAN, un 51\% contestaron afirmativamente, no así un $49 \%$ no. Lo que significa que casi la mitad de la población de bachilleres no conoce la oferta académica de la Universidad.

Relacionado a si les gustaría estudiar en la URACCAN, de manera general la mayoría de los y las estudiantes (77\%) respondió que SI les gustaría, un $23 \%$ dijeron que NO y un 0.09 no respondió. Con respecto a las demandas por los diferentes actores consultados podemos destacar la actualidad de nuestra oferta académica ya que todas las carreras siguen siendo de preferencia de estudios y profesionalización. 
Así mismo podemos resaltar que la población estudiantil además de reconocer y aceptar las carreras actuales que se oferta en los recintos y en las extensiones han considerado necesario dar apertura a nuevas carreras.

La demanda de formación por los y las estudiantes del décimo y undécimo grado, corresponde por Recinto a:

a. En los 4 recintos: Medicina, Derecho, Ingeniería en Sistema, Administración de Empresa, Ingeniería Civil.

b. En 3 recintos (Las Minas, Bilwi y Bluefields): Ingeniería Agroforestal.

c. En 2 recintos (Bilwi y Bluefields): Contabilidad, Sociología, Psicología y Enfermería.

d. En 1 recinto Nueva Guinea Medicina Veterinaria e inglés y en Bluefields Arquitectura.

Por otra parte las entidades de los territorios que fueron entrevistados dieron sus consideraciones con una perspectiva que está un poco orientada al plan de desarrollo de la costa y de acuerdo al contexto de su territorio. Con relación a la demanda de los empleadores se puede destacar el interés por la formación técnica así también por la oferta e implementación por parte de la Universidad de nuevas carreras.

De forma general podemos mencionar las carreras mas demandadas según los actores consultados por Recinto (cuadro 3).

Cuadro No. 3. Consolidado general de demanda de oferta académica por Recinto, 2013

\begin{tabular}{|l|l|}
\hline Recinto & Carreras mas demandadas \\
\hline & - Medicina. \\
& - Ingeniería civil. \\
& - Ingeniería en sistema. \\
Bluefields & - Admón. de Empresa. \\
& - Derecho. \\
& - Ing. Agroforestal. \\
& - Contabilidad. \\
& - Sociología. \\
& - Enfermería. \\
\hline
\end{tabular}




\begin{tabular}{|c|l|}
\hline Recinto & Carreras mas demandadas \\
\hline \multirow{5}{*}{ Bilwi } & - Medicina. \\
& - Ingeniería civil. \\
& - Ingeniería en sistema. \\
& - Admón. de Empresa. \\
& - Ing. Agroforestal. \\
& - Contabilidad. \\
& - Sociología. \\
& - Enfermería. \\
\hline \multirow{5}{*}{ Las Minas } & - Medicina. \\
& - Ingeniería civil. \\
& - Ingeniería en sistema. \\
& - Admón. de Empresa. \\
& - Derecho. \\
& - Ing. Agroforesta.l \\
\hline \multirow{5}{*}{ Nueva Guinea } & - Medicina. \\
& - Ingeniería civil. \\
& - Ingeniería en sistema. \\
& - Admón. de Empresa. \\
& - Derecho. \\
\hline
\end{tabular}

Como podemos observar en el cuadro anterior las carreras demandadas para ser ofertadas e implementadas en los cuatro Recintos son:

- Medicina.

- Ingeniería Civil.

- Ingeniería en Sistema.

- Derecho con énfasis en derecho indígena.

- Administración de Empresas.

\section{Pertinencia de la oferta académica actual en base al potencial de desarrollo de la Costa Caribe}

La Costa Caribe de Nicaragua con su diversidad étnica y profunda riqueza cultural ocupa aproximadamente el $46 \%$ del territorio nacional. Aún en las condiciones de histórica marginalización, representa para el país el 35\% del hato ganadero, 23\% del total del área agrícola, más del 80\% del área forestal, $70 \%$ de la producción pesquera, $60 \%$ de

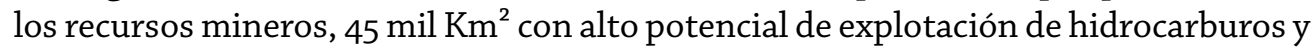
más de $700 \mathrm{Km}$. de costas en una de las zonas turísticas más reconocidas del mundo. 
Esta evidente combinación de riquezas naturales y humanas, historia, diversidad cultural y posición geográfica, debería asegurar condiciones de vida dignas a la población caribeña y hacer sustantivos aportes sociales, económicos y políticos al resto del país.

La Estrategia de Desarrollo de la Costa Caribe de Nicaragua evidencia la necesidad de construir, con la mayor urgencia posible, un Modelo de Desarrollo Humano Integral, en armonía con la cultura y la geografía del Caribe, que nos permita fortalecer la identidad cultural y aumentar el crecimiento económico con equidad.

En este sentido, plantea un marco estratégico de prioridades organizado en tres ejes que permita establecer las bases de un modelo de desarrollo con equidad que logre: (1) Incrementar el Bienestar Socioeconómico para la población del Caribe; (2) Alcance una Transformación Económica Equitativa, Sostenible y Armónica entre los seres humanos y la naturaleza; y (3) Fortalezca la Institucionalidad Autonómica para conducir el Desarrollo Humano.

En base a lo anterior la Universidad debe responder a las expectativas de la comunidad. En términos más amplios, a los aspectos referentes a las prioridades establecidas por los planes de desarrollo. Por lo que a continuación se detalla los ejes y programas de la Estrategia de Desarrollo de la Costa Caribe de Nicaragua versus las carreras que oferta la Universidad.

La Universidad debe ir revisando su oferta académica ya que si bien es cierto que se cubren algunas áreas en la formación de profesionales de la estrategia de desarrollo de la Costa Caribe otras quedan pendientes de ser trabajadas como: Ordenamiento territorial, seguridad alimentaria, agua y saneamiento, convivencia comunitaria y administración de justicia, protección de los bosques y los ecosistemas, Transporte (canal intercostero, construcción de carreteras), energía renovable y agua (hidroeléctricas) y Agroforestería comunitaria.

\section{El empleo en la Costa Caribe}

Según estadísticas del Instituto Nacional de Estadística y Censos (INEC 2005), la Población Económicamente Activa (PEA) de las Regiones Autónomas está representada por el $46.0 \%$ de personas potencialmente aptas para trabajar. El informe de gobierno del 2007 refleja un rango de empleos directos de 10-20\% para toda la RAAN. Por otro lado, algunos organismos no gubernamentales PRESANCA, 2006; Fundación para la Autonomía y Desarrollo de la Costa Atlántica de Nicaragua (FADCANIC, 2007), mencionan indistintamente cifras que van desde el 6o-80 \% el nivel de desempleo efectivo en la RAAN (Fundación para el Desarrollo Tecnológico Agropecuario y Forestal de Nicaragua, FUNICA, 2011). 
Los indicadores del mercado laboral provenientes del Ministerio del Trabajo (MITRAB) -tasa de participación, tasa de ocupación y tasa de desocupación- para las regiones autónomas muestra un comportamiento similar al promedio nacional como lo ilustran las gráficas adjuntas del MITRAB. Sin embargo hay algunas señales y percepciones de que dichos indicadores tendrían un comportamiento diferente si las encuestas lograsen captar la información proveniente de las comunidades alejadas de los centros urbanos donde suelen levantarse las encuestas (PNUD, 2005).

\section{El empleo de profesionales formados por la URACCAN}

En el año 2011, la Dirección Académica de la URACCAN realizó el Seguimiento a profesionales graduados de la URACCAN, generación 2001-2007, esta investigación se realizó con el objeto de valorar la formación en el nivel de licenciatura y el desempeño profesional de la cohorte generacional antes mencionada. En la investigación se encuestaron a 993 profesionales, distribuidos así: 290 de Las Minas, 253 de Nueva Guinea, 240 de Bilwi y 210 de Bluefields, de un total de 1132 profesionales de 19 programas de formación profesional. En los resultados se encontró que el 93\% de los profesionales se encuentran empleados, el $88.06 \%$ son asalariados y $4.94 \%$ son patrones o empleadores. De los asalariados, el 63\% laboran en instituciones del sector estatal donde ejercen funciones principalmente administrativas y de docencia (Castillo, et al. 2011).

\section{Conclusiones}

- A partir del año 2004 en la Costa Caribe nicaragüense existe la presencia de cuatro universidades: Universidad de las Regiones Autónomas de la Costa Caribe Nicaragüense (URACCAN), Bluefields Indian and Caribean University (BICU), Martin Lutero (UML), y Universidad de Occidente (UDO). Esta última, actualmente no tiene presencia en la región.

- Las áreas de conocimiento que han sido atendidas con la formación de profesionales con mayor cobertura en oferta de carreras son: Ciencias de la Educación, Humanidades, Ciencias Administrativas e Informática, Ciencias de la Salud. Con menor atención: Recursos Naturales y Medio Ambiente, Tecnología y construcción e Ingenierías Tecnológicas.

- Existen carreras que son comunes en las tres universidades mencionadas como: Educación Física, Enfermería, Licenciatura en Contabilidad Pública y Auditoría, Licenciatura en Ingles y Licenciatura en Pedagogía.

- Durante el período del 2002 al 2012, en la URACCAN se han ofertado veintiséis carreras, de las cuales, diez carreras han sido las más demandadas históricamente. Las cuales son:, Ingeniería Agroforestal, Licenciatura en Administración de Empresas, Licenciatura en Sociología con mención en Autonomía, Informática Administrativa, Ingeniería en Zootecnia, Licenciatura en Ciencias Sociales con mención en Desarrollo Local, Licenciatura en Ciencias 
de la Educación mención en español, Licenciatura en Ciencias de la Educación mención en Biología, Licenciatura en Contabilidad Pública y Auditoría y Licenciatura en Pedagogía.

- En el período 2002 al 2012 hubo un incremento significativo en la demanda de siete carreras: Licenciatura en Administración de Empresas, Ingeniería Agroforestal, Licenciatura en Sociología con mención en Autonomía, Licenciatura en Pedagogía, Licenciatura en Contabilidad Pública y Auditoría, Licenciatura en Ciencias de la Educación con mención en Biología y Licenciatura en Informática Administrativa.

- La mayoría de la población estudiantil del nivel de secundaria básica (futuros bachilleres) de la Costa Caribe le gustaría estudiar en la URACCAN, aunque el $49 \%$ no conoce la oferta académica.

- Las carreras más demandadas por los diferentes actores para ser ofertadas e implementadas en los cuatro Recintos son: Medicina, Ingeniería Civil, Ingeniería en Sistema, Derecho con énfasis en Derecho Indígena y Administración de Empresas. En los tres Recintos de Las Minas, Bilwi y Bluefields: Ingeniería Agroforestal. Demandas por dos de los Recintos de Bilwi y Bluefields: Contabilidad Pública y Auditoría, Sociología con mención en Autonomía, Contabilidad Pública y Auditoría y Enfermería. Es importante resaltar la demanda en Bluefields de: Ingeniería Naval, Ingeniería Industrial, Lic. en Náutica y transporte marítimo (Navegación).

- La oferta académica de la Universidad cubre algunas áreas en la formación de profesionales de la estrategia de desarrollo de la Costa Caribe, pero otras quedan pendientes de ser trabajadas como: ordenamiento territorial, seguridad alimentaria, agua y saneamiento, convivencia comunitaria y administración de justicia, protección de los bosques y los ecosistemas, Transporte (canal intercostero, construcción de carreteras), energía renovable y agua (hidroeléctricas) y Agroforestería comunitaria.

- La población costeña vive de la agricultura, seguido del sector de servicios, de la pesca y del comercio por lo que la reorientación de la oferta académica de la URACCAN debe considerar estos sectores. 


\section{Lista de referencia}

Alejo, S; Martí, M; Ruiz, G. (2009). La oferta y la demanda educativa: comportamiento del egreso y admisión de las preparatorias de la universidad de Guanajuato. EDUCATIO 8, México.

Castillo, L; Aragón, M; García, A; Dávila A; Baltodano, M. (2011). Seguimiento a profesionales graduados de la URACCAN, generación 2001-2007. URACCAN. Managua Nicaragua. Revista Ciencia e Interculturalidad. 8 (1):11-24.

Corrales Munguia, E. Guido Pichardo M, Calderón Chávez, Claudia y Herdocia Balladares, Cecilia (2004). Diagnóstico situacional sobre las características de las instituciones de Educación Superior nicaragüenses legalmente establecidas. CNU, Managua, Nicaragua.

FUNICA. 2009. Caracterización socioeconómica de la Región Autónoma del Atlántico Norte (RAAN) de Nicaragua. Managua, Nicaragua.

PNUD. 2005. Informe de desarrollo humano 2005. Las regiones autónomas de la Costa Caribe. ¿Nicaragua asume su diversidad? PNUD. Managua Nicaragua. Pag. 155-157.

PNUD. 2011. Informe nacional sobre desarrollo humano 2011. Las juventudes construyendo Nicaragua. PNUD. Managua Nicaragua. Pag. 145.

UDUAL (2007). Educación Superior en América Latina y el Caribe: Sus estudiantes hoy. Primera edición. Union de Universidades de América Latina y el Caribe, México.

URACCAN (2004). Modelo Pedagógico.

URACCAN (2012). Régimen Académico. 